\title{
Decreased Splenic CD4 ${ }^{+}$T-Lymphocytes in Apolipoprotein M Gene Deficient Mice
}

\author{
Zhigang Wang, ${ }^{1}$ Guanghua Luo, ${ }^{2}$ Yuehua Feng, ${ }^{2}$ Lu Zheng, ${ }^{2}$ Hongyao Liu, ${ }^{1}$ Yun Liang, \\ Zhonghua Liu, ${ }^{1}$ Peng Shao, ${ }^{1}$ Maria Berggren-Söderlund, ${ }^{3}$ Xiaoying Zhang, ${ }^{1}$ and Ning Xu${ }^{3}$ \\ ${ }^{1}$ Department of Cardiothoracic Surgery, Third Affiliated Hospital of Soochow University, Changzhou 213003, China \\ ${ }^{2}$ Comprehensive Laboratory, Third Affiliated Hospital of Soochow University, Changzhou 213003, China \\ ${ }^{3}$ Division of Clinical Chemistry and Pharmacology, Department of Laboratory Medicine, Lund University, 22185 Lund, Sweden \\ Correspondence should be addressed to Xiaoying Zhang; zhangxy6689996@163.com and Ning Xu; ning.xu@med.lu.se
}

Received 16 July 2015; Revised 23 September 2015; Accepted 28 September 2015

Academic Editor: Fabrizio Montecucco

Copyright (C) 2015 Zhigang Wang et al. This is an open access article distributed under the Creative Commons Attribution License, which permits unrestricted use, distribution, and reproduction in any medium, provided the original work is properly cited.

\begin{abstract}
Spleen T-lymphocytes, especially $\mathrm{CD} 4^{+}$T-cells, have been demonstrated to be involved in broad immunomodulation and hostdefense activity in vivo. Apolipoprotein $\mathrm{M}$ gene (apoM) may have an important role in the regulation of immunoprocess and inflammation, which could be hypothesized to the apoM containing sphingosine-1-phosphate (S1P). In the present study we

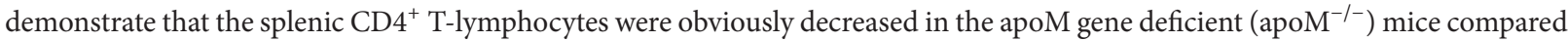
to the wild type $\left(\mathrm{apoM}^{+/+}\right)$. Moreover, these mice were treated with lipopolysaccharide (LPS) and it was found that even more pronounced decreasing $\mathrm{CD} 4^{+} \mathrm{T}$-lymphocytes occurred in the spleen compared to the apo $\mathrm{M}^{+/+}$mice. The similar phenomena were found in the ratio of $\mathrm{CD}^{+} / \mathrm{CD}^{+}$T-lymphocytes. After administration of LPS, the hepatic mRNA levels of tumor necrosis factor$\alpha$ (TNF- $\alpha)$ and monocyte chemotactic protein-1 (MCP-1) were markedly increased; however, there were no statistical differences observed between apo $\mathrm{M}^{+/+}$mice and apoM ${ }^{-/-}$mice. The present study demonstrated that apoM might facilitate the maintenance of $\mathrm{CD}^{+}$T-lymphocytes or could modify the T-lymphocytes subgroups in murine spleen, which may further explore the importance of apoM in the regulation of the host immunomodulation, although the detailed mechanism needs continuing investigation.
\end{abstract}

\section{Introduction}

The apolipoprotein $\mathrm{M}$ gene (apoM), located on the major histocompatibility complex class III (MHC-III) region of chromosome 6p21.3, encodes a $26 \mathrm{Da}$ glycoprotein of 188 amino acids [1]. Many genes in this region are related to the immune and inflammatory response $[2,3]$. apoM is required for the formation of pre $\beta$-HDL particles and its presence in high-density lipoprotein (HDL) and pre $\beta$-HDL contributes to cellular cholesterol efflux in mice and humans [4]. It has been demonstrated that HDL could be a relevant player in both innate immunity and adaptive immunity. Such effects were explored mainly depending on the ability to finely modulate cholesterol bioavailability in immune cell $[5,6]$. Moreover, the beneficial effects of HDL on the immune inflammation in vivo could also depend on the cargo of proteins and lipids carried by HDL which confer additional functions [7].
Recent investigations indicate that apoM is a natural carrier of sphingosine-1-phospate (S1P) in vivo, an important bioactive lipid mediator known to be associated with HDL [8]. S1P is a bioactive lysophospholipid with multiple effects on angiogenesis, lymphocyte trafficking, endothelial cell migration, and inflammation [9]. The activation of S1P receptor 1 (S1PR1) and the resulting signaling cascade could facilitate the egress of T-lymphocytes from lymphoid organs and modulate T-lymphocyte lineage [10, 11]. Moreover, S1PR1 is a switch factor that inhibits the development of antiinflammatory regulatory $\mathrm{T}$ cells but drives differentiation of proinflammatory T helper type 1 cells (Th1) [12]. Previous investigations suggested apoM may also be involved in the inflammatory reaction and the potential immune-reactive properties [13-15].

The important role of apoM in modulating the inflammatory and immune response remains to be elucidated. With regard to immune function, the mammalian spleen plays 


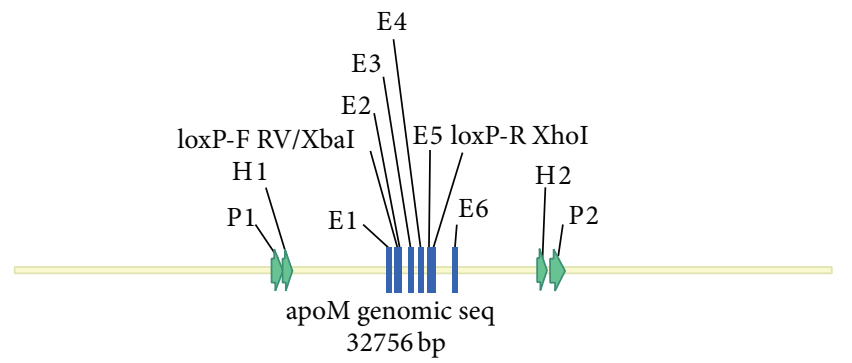

(a)

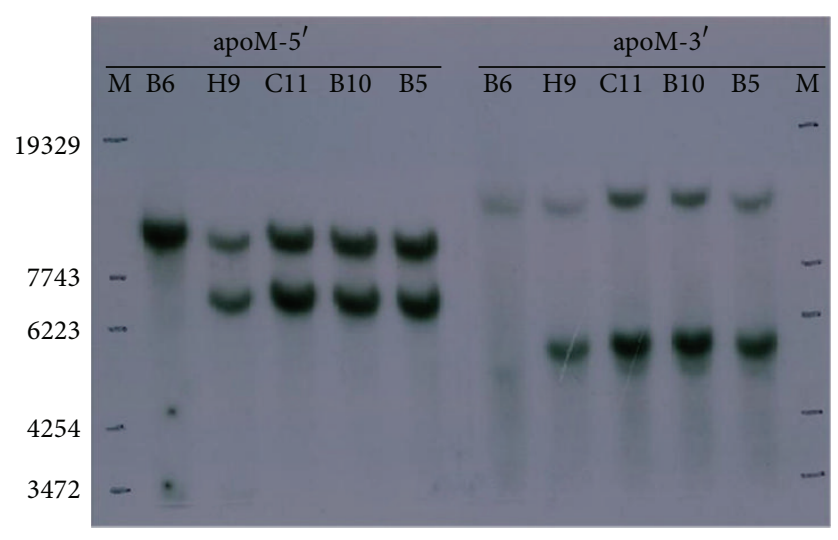

(c)
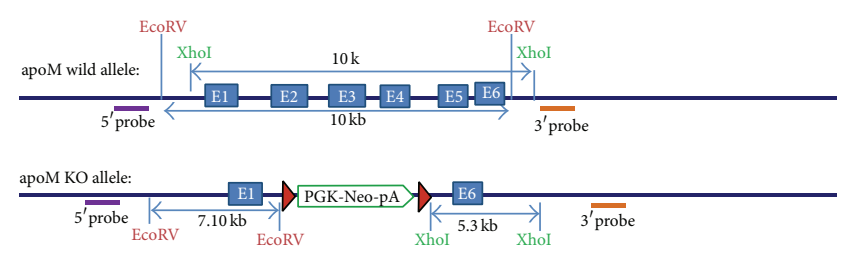

\begin{tabular}{lccc}
\hline & $\begin{array}{c}\text { Restriction } \\
\text { enzyme }\end{array}$ & Wild type & Targeted \\
\hline apoM-5 & EcoRV & $10 \mathrm{k}$ & $7.10 \mathrm{k}$ \\
apoM-3 & XhoI & $10 \mathrm{k}$ & $5.3 \mathrm{k}$ \\
\hline
\end{tabular}

Restriction enzyme and fragment size of southern blot

(b)

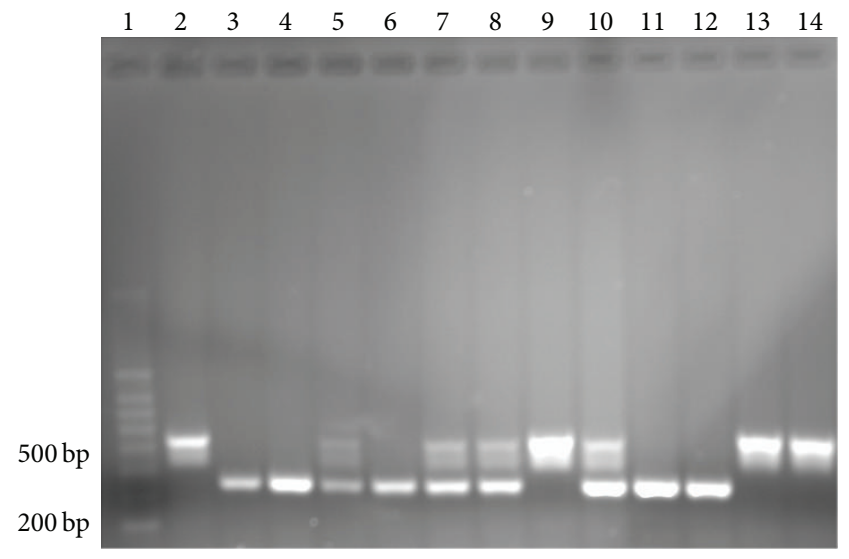

(d)

FIGURE 1: Generation of apoM deficient mice. (a) Replace apoM with a loxP-Neo-loxP cassette. (b) Southern blot analysis of $5^{\prime}$ and $3^{\prime}$ probes. (c) Results of Southern blot analyses of ES cells B5, H9, B10, and C11. B6 genomic DNA as negative control. (d) Genotyping generations of heterozygote/heterozygote and wild type crosses were performed by PCR of mouse tail DNA. Lanes 2, 9, 13, and 14 only showed bands at $558 \mathrm{bp}$, which was identified as apoM gene wild type; lanes 3, 4, 6, 11, and 12 only showed bands at $342 \mathrm{bp}$, which was identified as apoM gene deficient homozygotes; lanes 5, 7, 8, and 10 showed both 342 and 558 bp bands, which were identified as apoM gene heterozygotes.

important roles in the innate and adaptive immune systems. Analysis of the lymphocyte subgroups during development of inflammation could give important information on cell modifications occurring in the splenic lymphatic bed [16]. In addition, cytokines are immune regulators that were historically thought to be products solely of peripheral immune system. In the present study, we took advantage of wild type $\left(\right.$ apoM ${ }^{+/+}$) and apoM deficient (apoM ${ }^{-/-}$) mice to investigate whether the apoM affects the T-lymphocytes subgroups in the spleen with or without challenge of lipopolysaccharide (LPS). Additionally, we examined serum concentration and liver tissue mRNA levels of tumor necrosis factoralpha (TNF-alpha) and monocyte chemotactic protein-1 (MCP-1).

\section{Materials and Methods}

2.1. Generation of apoM Gene Deficient Mice. The apoM ${ }^{-/-}$ mice were generated by homologous recombination with the help of Model Animal Research Center of Nanjing University (China). Briefly, BAC-retrieval methods were used to construct the targeting vectors. apoM gene was retrieved from a C57BL/6 BAC clone by using a retrieval vector that contained two homologous arms. After correct recombination, this vector contained $3.2 \mathrm{~kb}$ of the genomic sequence, including exon I-VI and $5 \mathrm{~kb}$ downstream sequences. Then, the entire exon II-V of apoM was deleted and replaced with a loxP-Neo-loxP cassette (Figure 1(a)). The neocassette was in the reverse-transcriptional orientation compared with the apoM gene. The targeting construct, which contained a neomycin-expression cassette for positive selection and a herpes simplex virus-thymidine kinase expression cassette for negative selection, was linearized with the NotI restriction enzyme and electroporated into C57BL/6N-derived B6/BLU ES cells. ES cell clones (192) were selected and verified for correct recombination using long-range PCR and Southern blot analysis (Figures 1(b) and 1(c)). Correctly targeted ES cells were injected into $\mathrm{C} 57 \mathrm{BL} / 6$ blastocysts and transferred to pseudopregnant mice. Chimeric male mice were identified using PCR and were bred to C57BL/ 6 female mice to generate F1 offspring. The F1 offspring were crossed at least 2-3 generations of inheritance before the experiment. Subsequent genotyping generations of heterozygote/heterozygote and wild type crosses were performed by PCR of mouse tail DNA 
TABLE 1: Sequences of primers and probes.

\begin{tabular}{lcc}
\hline Gene & Primer/probe & Sequence $\left(5^{\prime}-3^{\prime}\right)$ \\
\hline TNF-alpha & Froward primer & tgacaagcctgtagcccacg \\
& Reverse primer & ttgtctttgagatccatgccg \\
MCP-1 & Probe & FAM-cgtagcaaaccaccaagtggaggagc-TAMRA \\
& Froward primer & gctggagagctacaagaggatcac \\
ccttcttggggtcagcacag
\end{tabular}

by using the common upstream primer $5^{\prime}$-GCCCAGACATGAAAACAGACCT- $3^{\prime}$ and downstream primers $5^{\prime}$-CCACTCCCACTGTCCTTTCCTAAT- $3^{\prime}$ and $5^{\prime}$-GGTACCATTCTAGCCCATAAGAATTAG-3', which generated 558 and 342 bp bands from the apoM $\mathrm{M}^{+/+}$and apoM $^{-/-}$alleles, respectively (Figure 1(d)).

2.2. Animals and Experimental Procedure. Age- and body weight-matched male $\mathrm{C} 57 \mathrm{BL} / 6$ apoM $^{+/+}$and apoM ${ }^{-/-}$mice (23-25 g) at 6-8 weeks were used in the present study. The mice were housed in specific pathogen-free conditions and a temperature and humidity controlled environment (12 hrs light/dark cycle) with unlimited access to tap water and food. All the animal work was conducted in compliance with the recommendations on the guide for the care and use of laboratory animals and approved by the local ethical committee.

Mice were randomly assigned to receive intraperitoneal (i.p.) injection of the vehicle (pyrogen-free $0.9 \%$ saline; control group), $5 \mathrm{mg} / \mathrm{kg}$ LPS (LPS; Escherichia coli serotype O111:B4; Sigma-Aldrich, St. Louis, MO), or $10 \mathrm{mg} / \mathrm{kg}$ LPS for three times every $24 \mathrm{hrs}$. All mice were sacrificed at $96 \mathrm{hrs}$ after the first i.p. injection. The blood samples and tissue samples were collected for further analysis. The spleens were prepared for the flow cytometry analyses immediately. The blood sample was centrifuged $\left(12,000 \times \mathrm{g}, 4^{\circ} \mathrm{C}, 20 \mathrm{~min}\right)$ and the serum was stored at $-80^{\circ} \mathrm{C}$ until assay. All other tissues were snap-frozen in liquid nitrogen and stored at $-80^{\circ} \mathrm{C}$ until processing.

2.3. Cell Preparation and Flow Cytometry Analysis. Spleens of the control group or LPS-challenged apoM $\mathrm{M}^{+/+}$and apoM $^{-/-}$ mice were harvested aseptically and filtered through cell strainers to produce a single-cell suspension. After lysis of red blood cells, the spleen suspensions were washed three times with PBS and adjusted to a concentration of $1.0 \times 10^{7} / \mathrm{mL}$. To detect the changes in lymphocyte subgroups, the prepared splenocytes $\left(1.0 \times 10^{7} / \mathrm{mL}, 100 \mu \mathrm{L}\right)$ were incubated with $0.5 \mu \mathrm{L}$ each of fluorescently labeled antibodies for 30 minutes at $4^{\circ} \mathrm{C}$ protected from light and washed three times with PBS before analysis. Fluorescently labeled antibodies included FITC-conjugated anti-CD3, PECy5 conjugated anti-CD4, and PE-Cy7 conjugated anti-CD8 (BioLegend, San Diego, CA). The stained cells were then analyzed by flow cytometry immediately (FC500, Beckman, USA).

2.4. Determinations of $m R N A$ Levels of TNF-Alpha and MCP1 in Livers. Total RNA was isolated from the liver tissue by the guanidinium thiocyanate method, and the quality of the samples was determined by the absorbance measurements at $260 / 280 \mathrm{~nm}$. $2 \mu \mathrm{g}$ total RNA was reverse-transcribed to cDNA, using the first strand cDNA synthesis kit (Qiagen) according to the manufacturer's instructions. The mRNA levels were measured under real-time PCR using TaqMan assay for both target and reference genes. Mouse TNF-alpha and MCP-1 primer/probe sets were designed according to the information of GenBank, as presented in Table 1. GAPDH was used in separate tubes as reference control. Relative standard curves for all three templates were performed to compensate the efficiency of the PCRs. Quantification of TNF-alpha or MCP-1 mRNA levels was relative to the GAPDH mRNA levels. The real-time PCR reaction was performed on LightCycler 480 in a final volume of $25 \mu \mathrm{L}$. Each well of a 96-well plate was containing $2.5 \mu \mathrm{L} 10 \times$ PCR buffer, $1.5 \mu \mathrm{L} \mathrm{MgCl} 2$ (25 mM), $0.5 \mu \mathrm{L}$ dNTPs $(10 \mathrm{mM})$, $0.25 \mu \mathrm{L}$ Taq DNA polymerase, $0.1 \mu \mathrm{L} 100 \mu \mathrm{M}$ each primer and probe, $2 \mu \mathrm{L} \mathrm{cDNA}$, and ddH2O $17.95 \mu \mathrm{L}$. Thermal cycling conditions for TNF-alpha and MCP-1 included the following steps: denaturation at $95^{\circ} \mathrm{C}$ for $3 \mathrm{~min}$, followed by 40 cycles at $98^{\circ} \mathrm{C}$ for $5 \mathrm{~s}$ and $60^{\circ} \mathrm{C}$ for $27 \mathrm{~s}$ (for $\mathrm{GAPDH}, 60^{\circ} \mathrm{C}$ for $15 \mathrm{~s})$.

2.5. Determinations of TNF-Alpha and MCP-1 in Serum. Based on the method of Luminex technology [17], the serum concentrations of TNF-alpha and MCP-1 were determined by using MILLIPLEX xMAP Mouse Cytokine/Chemokine Kit (Merck Millipore, USA). The procedure was performed according to the manufacturer's protocol. Quantification of these cytokines was performed using the Luminex 200 (Luminex Corp., USA) platform.

2.6. Statistics. Data are expressed as median with range. Statistical analyses were performed with the GraphPad Prism 6.0 software (GraphPad Software, Inc., San Diego, California, USA). Multiple comparisons were performed with one-way ANOVA, followed by Dunnett's multiple comparison test analysis. And comparisons between two 


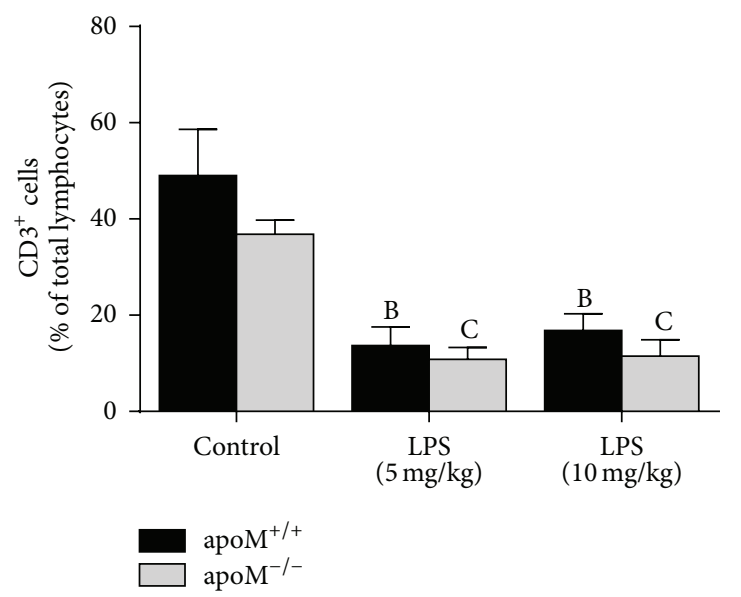

(a)

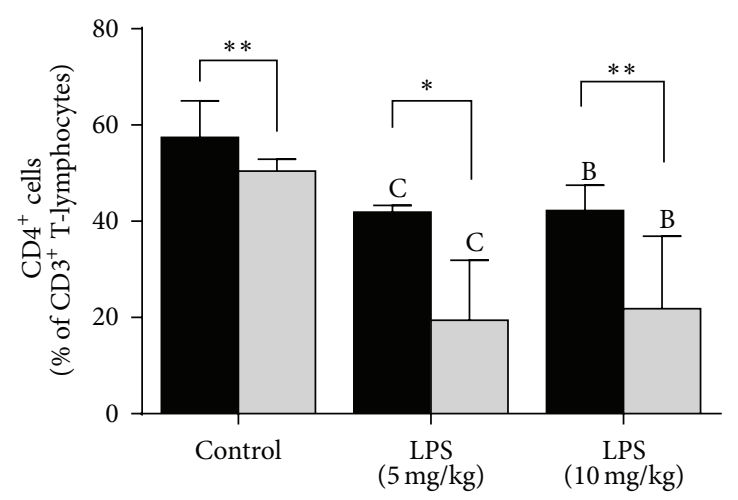

apoM $\mathrm{M}^{+/+}$ $\mathrm{apoM}^{-/-}$

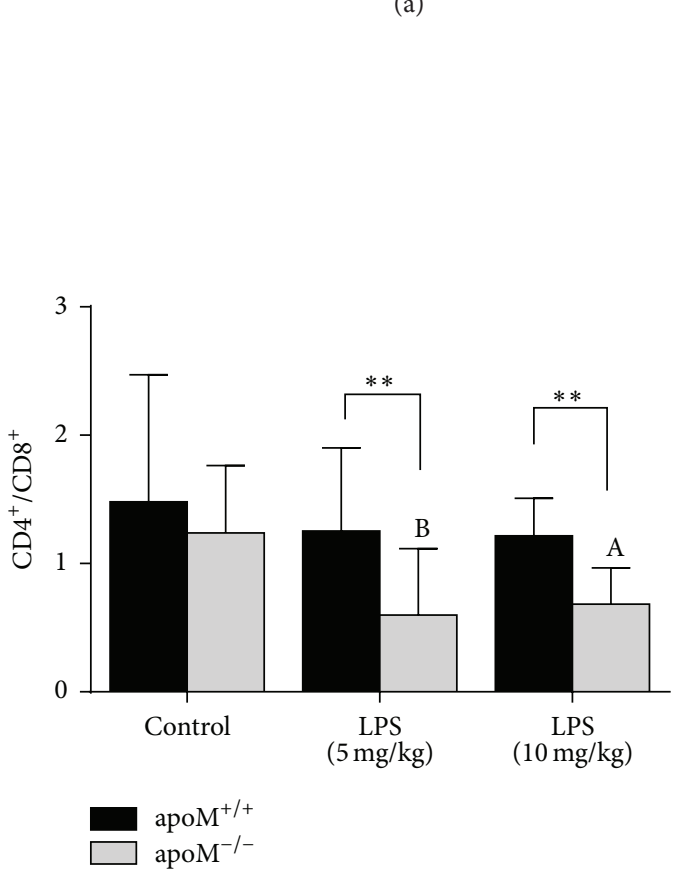

(c)

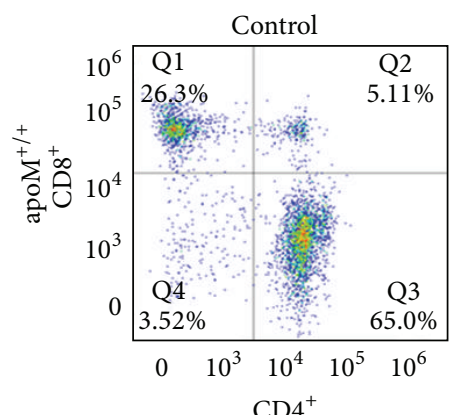

(b)

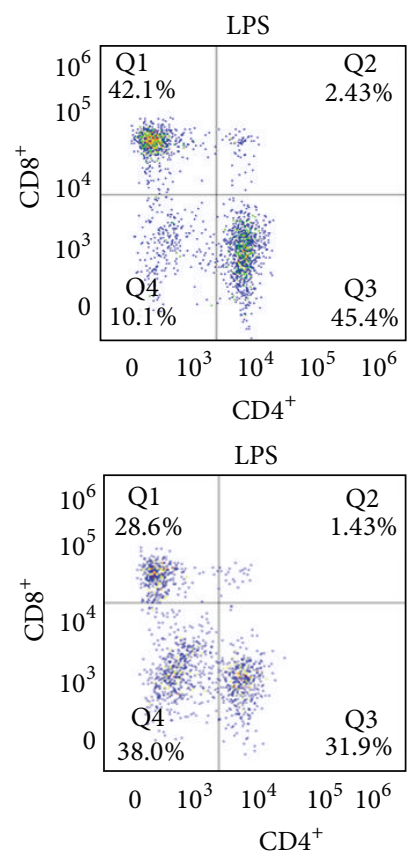

(d)

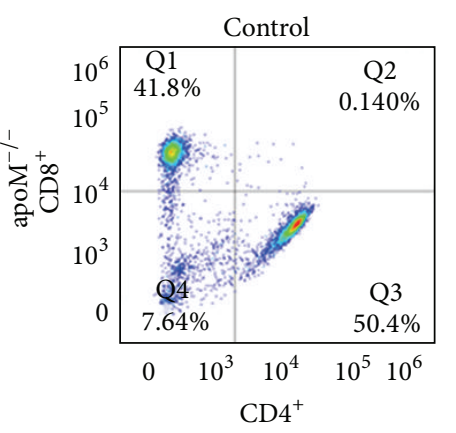

Figure 2: Distribution of splenic T-lymphocyte and its subpopulations in apoM $\mathrm{M}^{-/-}$and apoM ${ }^{+/+}$mice with or without administration of

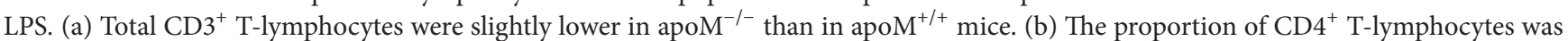
obviously lower in apoM ${ }^{-/-}$mice than that in apoM $\mathrm{M}^{+/+}$mice before LPS treatment. After administration of LPS, the CD4 $4^{+} \mathrm{T}-$ lymphocytes were dramatically decreased in the apoM ${ }^{-/-}$mice, whereas $\mathrm{CD} 4^{+}$T-lymphocytes were only moderately decreased. (c) The ratio of CD $4^{+} / \mathrm{CD} 8^{+}$ was also obviously decreased in apoM ${ }^{-/-}$mice compared to that in $\mathrm{apoM}^{+/+}$mice after LPS treatment. (d) Representative FACS plots of splenic $\mathrm{CD}^{+}$and $\mathrm{CD}^{+}$T-lymphocytes in the apoM ${ }^{+/+}$and apoM ${ }^{-/-}$mice $\left(n=5 \sim 6\right.$, one-way ANOVA followed by Dunnett's test, ${ }^{\mathrm{A}} p<0.05$, ${ }^{\mathrm{B}} p<0.01$, and ${ }^{\mathrm{C}} p<0.001$ versus apoM $\mathrm{M}^{+/+}$or apoM ${ }^{-/-}$control; Mann-Whitney $U$ test, ${ }^{*} p<0.05$ and $\left.{ }^{* *} p<0.01\right)$.

groups were statistically evaluated by two-tailed MannWhitney $U$ test. $p$ values less than 0.05 were considered significant.

\section{Results}

3.1. The Splenic Total T-Lymphocytes $\left(\mathrm{CD}^{+}\right.$Cells) and T-Lymphocyte Subgroups $\left(\mathrm{CD}^{+}\right.$and $\mathrm{CD}^{+}$Cells) Were Determined by Flow Cytometry. As shown in Figure 2(a), the total CD3 ${ }^{+}$ T-lymphocytes were slightly lower in the apoM ${ }^{-/-}$mice than those in apoM $\mathrm{M}^{+/+}$mice; after administration of LPS, the total $\mathrm{CD}^{+}$T-lymphocytes were dramatically decreased in both apoM ${ }^{-/-}$mice and apoM ${ }^{+/+}$mice $(p<0.001$ and $p<0.01$, resp.), although no statistical differences occurred in the apoM gene deficient mice compared to the wild type. Interestingly, the basal proportion of $\mathrm{CD} 4^{+} \mathrm{T}$-lymphocytes in apoM $^{-/-}$mice was significantly lower than that in apoM $^{+/+}$ mice (Figure 2(b), $p<0.01$ ). After administration of LPS, at both concentrations of $5 \mathrm{mg} / \mathrm{kg}$ and $10 \mathrm{mg} / \mathrm{kg}$, the proportion of $\mathrm{CD}^{+}$T-lymphocytes was significantly decreased in apoM $^{-/-}$and apoM ${ }^{+/+}$mice (Figure 2(b), both $p<0.001$ ). Furthermore, the decrease of $\mathrm{CD}^{+}$T-lymphocytes in 


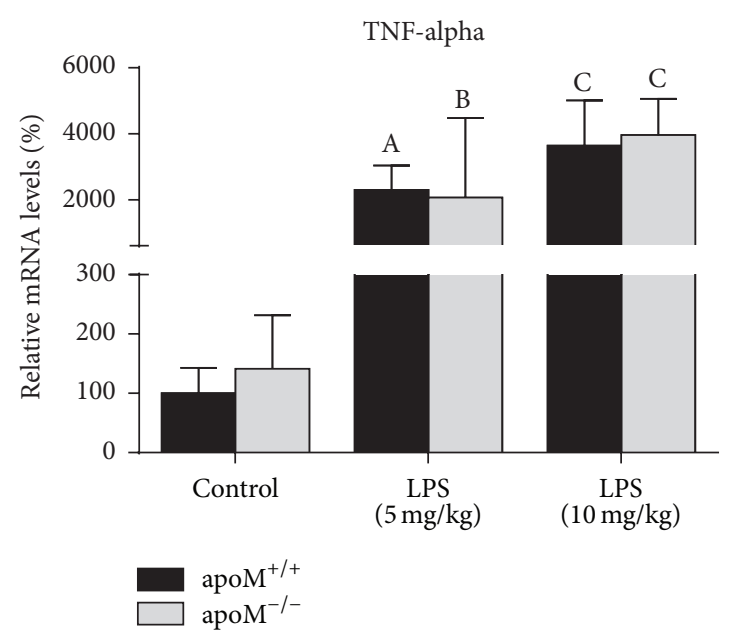

(a)

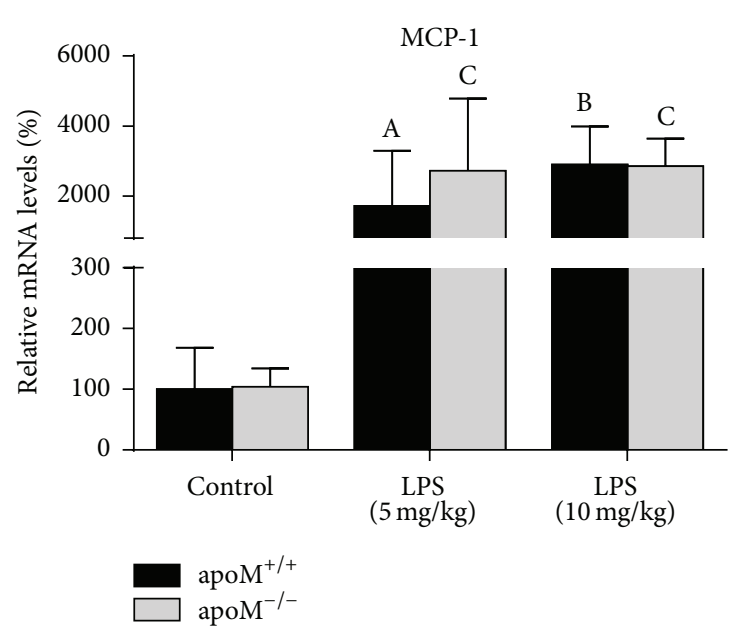

(b)

FIGURE 3: Hepatic mRNA levels of TNF-alpha and MCP-1 in apoM $\mathrm{M}^{-/-}$mice and apoM ${ }^{+/+}$mice with or without LPS treatment. The mRNA levels of TNF-alpha (a) and MCP-1 (b) were similar in apoM ${ }^{+/+}$mice and apoM ${ }^{-/-}$mice before LPS administration. After LPS treatment the hepatic mRNA levels of TNF-alpha and MCP-1 were dramatically increased in both apoM $\mathrm{M}^{+/+}$and apoM ${ }^{-/-}$mice. The mRNA levels in apoM $^{+/+}$mice in the control group were considered as $100 \%\left(n=6 \sim 9\right.$, one-way ANOVA followed by Dunnett's test, ${ }^{\mathrm{A}} p<0.05,{ }^{\mathrm{B}} p<0.01$, and ${ }^{\mathrm{C}} p<0.001$ versus apoM $\mathrm{M}^{+/+}$or apoM ${ }^{-/-}$control).

apoM $^{-/-}$mice was much more than that in apoM ${ }^{+/+}$mice $(p<0.05$ and $p<0.01$, resp.). There was no significant difference for the splenic $\mathrm{CD}^{+} \mathrm{T}$-lymphocytes between apoM $\mathrm{M}^{+/+}$ and apo $^{-1-}$ mice in the control group and LPSchallenged mice (data not show). As shown in Figure 2(c), the similar phenomena with $\mathrm{CD}^{+}$T-lymphocytes were found in the ratio of $\mathrm{CD} 4^{+} / \mathrm{CD}^{+}$T-lymphocytes, although there was only slight decrease of basal $\mathrm{CD} 4^{+} / \mathrm{CD}^{+}$ratio in apoM $^{-/-}$mice compared to apoM ${ }^{+/+}$mice $(p=0.0556)$.

\subsection{The Hepatic mRNA Levels and Serum Protein Levels} of TNF-Alpha and MCP-1 in apoM ${ }^{+/+}$and apoM-l- Mice Were Determined by Real-Time PCR or Luminex Technology, Respectively. As shown in Figure 3, the hepatic mRNA levels of TNF-alpha and MCP-1 were significantly increased in both apoM $\mathrm{M}^{+/+}$and apoM $\mathrm{M}^{-/-}$mice after administration of LPS, whereas there were no statistical differences between apoM $^{-/-}$mice and apoM ${ }^{+/+}$mice. The serum levels of TNFalpha in both apoM $\mathrm{M}^{+/+}$and apoM $^{-/-}$mice significantly increased after LPS administration (Figure 4(a), $p<0.001$ and $p<0.05$, resp.). However, the serum levels of TNF-alpha had no statistical difference between apoM $\mathrm{M}^{+/+}$and apoM ${ }^{-/-}$ mice in the control group and $5 \mathrm{mg} / \mathrm{kg}$ LPS-treated mice. The serum levels of TNF-alpha in apoM ${ }^{-/-}$mice were extremely lower after $10 \mathrm{mg} / \mathrm{kg}$ LPS stimulation compared to apoM $\mathrm{M}^{+/+}$ mice $(p<0.01)$. The serum levels of MCP-1 in both apoM ${ }^{+/+}$ and apoM $^{-/-}$mice were significantly increased after LPS administration (Figure 4(b), $p<0.01$ and $p<0.05$, resp.). The serum levels of MCP-1 in apoM ${ }^{-/-}$mice were moderately higher than those in apoM ${ }^{-/-}$mice $(p<0.05)$, while no statistical differences were observed between $\operatorname{apoM}^{+/+}$and apoM $^{-/-}$mice after LPS treatment.

\section{Discussion}

$\mathrm{CD}^{+}{ }^{+}$T-lymphocytes, also known as T helper cells, play an important role in orchestrating adaptive immune responses to various microbes. $\mathrm{CD} 4^{+}$T-lymphocytes recognize peptide-major histocompatibility complex (MHC) class II complexes presented by antigen-presenting cells (APCs) and differentiate into at least four lineages, Th1, Th2, Th17, and inducible $\mathrm{T}$ regulatory cells, which participate in different types of immune responses [18]. As a Toll-like receptor (TLR) 4 agonist, LPS stimulates TLR 4 and then activates antigenpresenting cells through MyD88- and TRIF-dependent signaling pathways [19], which activates nuclear factor(NF-) $\kappa \mathrm{B}$, leading to release of inflammatory cytokines, and initiates $\mathrm{CD}^{+}$T-lymphocytes clonal expansion and functional differentiation [20].

apoM is the physiological carrier protein of S1P in HDL and apoM can deliver S1P to the S1PR1 [8], which can affect mammalian immunity through alterations of thymocyte emigration, differentiation of T-lymphocytes subgroups, and lymphocyte trafficking in lymphoid organs and other tissues [21]. It has been reported that HDL can promote the interaction of LPS with LPS-binding protein (LBP) favoring LPS clearance $[22,23]$, whereas the genetic knock-out apoM is associated with the absence of pre $\beta$-HDL particles and reduction of S1P in serum $[4,8]$.

In the present study, we demonstrated that the splenic $\mathrm{CD}^{+}$T-lymphocytes significantly decreased, whereas no obvious changes occurred on the $\mathrm{CD}^{+} \mathrm{T}$-lymphocytes in apoM $^{-/-}$mice compared to apoM ${ }^{+/+}$mice. After administration of LPS, total CD3 ${ }^{+}$T-lymphocytes were significantly decreased in both apoM ${ }^{-/-}$and apoM $^{+/+}$mice, although there were no statistical differences between $\mathrm{apoM}^{-/-}$and apoM $^{+/+}$mice. However, the splenic proportion of $\mathrm{CD}^{+}$ 


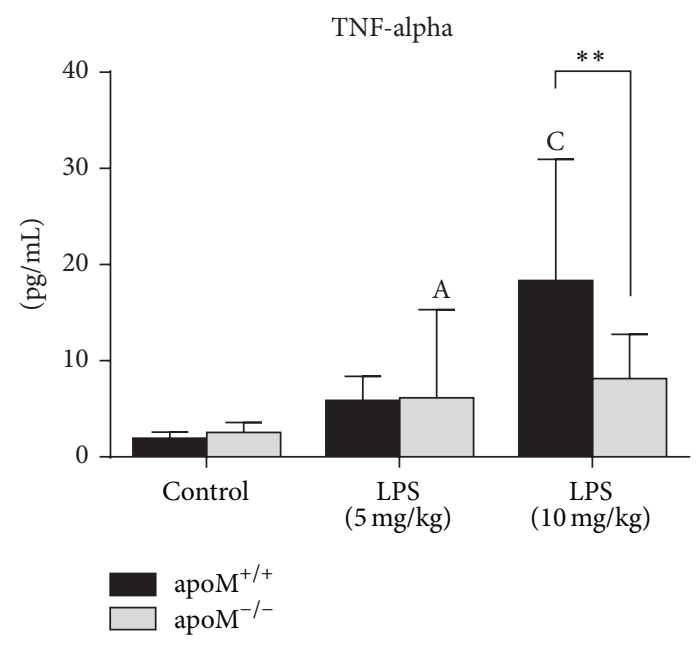

(a)

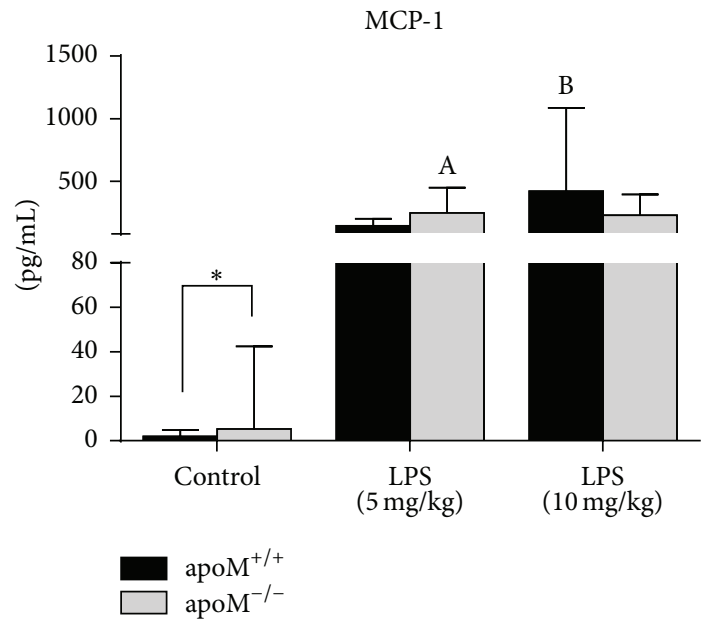

(b)

FIGURE 4: Serum protein levels of TNF-alpha and MCP-1 in apoM ${ }^{-/-}$mice and apoM ${ }^{+/+}$mice with or without LPS treatment. (a) The serum levels of TNF-alpha in both apoM $\mathrm{M}^{+/+}$and apoM ${ }^{-/-}$mice were significantly increased after LPS treatment. (b) The basal MCP-1 levels were higher in apoM $\mathrm{M}^{-/-}$mice than in apoM $\mathrm{M}^{+/+}$mice. After LPS treatment, serum levels of MCP-1 were dramatically increased in both apoM ${ }^{+/+}$ and apoM ${ }^{-/-}$mice, but there were no statistical differences between apoM ${ }^{-/-}$mice and apoM ${ }^{+/+}$mice $(n=6$, one-way ANOVA followed by Dunnett's test, ${ }^{\mathrm{A}} p<0.05,{ }^{\mathrm{B}} p<0.01$, and ${ }^{\mathrm{C}} p<0.001$ versus apoM ${ }^{+/+}$or apoM ${ }^{-/-}$control; Mann-Whitney $U$ test, ${ }^{*} p<0.05$ and $\left.{ }^{* *} p<0.01\right)$.

T-lymphocytes was dramatically decreased in apoM ${ }^{-/-}$ mice and there was only moderate decrease of $\mathrm{CD}^{+}{ }^{-} \mathrm{T}$ lymphocytes in apoM $^{+/+}$mice (Figure 2(b)). The similar phenomenon was found for the ratio of $\mathrm{CD} 4^{+} / \mathrm{CD} 8^{+}$in apoM $^{-/-}$mice (Figure 2(c)). These findings coupled with systemic inflammation and sepsis challenged by LPS caused marked lymphopenia that resulted in the decrease of total splenic T-lymphocytes and $\mathrm{CD} 4^{+} / \mathrm{CD}^{+} \mathrm{T}$-lymphocytes ratio [24]. It has been previously reported that apoA-I, a major protein contained in HDL, is essential for HDL formation and function. With lipid-free apoA-I injection, male $\mathrm{LDLr}^{-/-}$ and apoA- $\mathrm{I}^{-/-}$DKO mice exhibited reduced infiltrates and inflammation in skin and lymph nodes compared with $\mathrm{LDLr}^{-/-}$SKO mice, including an increase in regulatory $\mathrm{T}$ cell expansion within the $\mathrm{CD} 4^{+}$T-lymphocytes pool [25]. The genetic approaches to alter the function of S1PR1 have established that S1PR1 is the main S1P receptor that regulated T-lymphocytes trafficking: T-lymphocytes from S1PR1deficient mice failed to egress from the thymus and peripheral lymphoid organs, whereas S1PR1-transgenic T-lymphocytes preferentially distributed to the blood rather than lymphoid organs [26]. apoM-S1P is able to activate the S1PR1 and affect the function of endothelial cells [8]. Meanwhile apoM-1mice with the congenital low S1P level impaired the ability of activating S1PR1 in the endothelial cells, which may reduce the trafficking of T-lymphocyte, especially $\mathrm{CD} 4^{+} \mathrm{T}$ lymphocytes, between the thymus and the spleen, and result in the decrease of splenic $\mathrm{CD} 4^{+}$T-lymphocytes or the $\mathrm{CD} 4^{+}$ T-lymphocytes would distribute to the blood rather than the spleen. A recent paper published in Nature [27] demonstrated that the absolute counting of $\mathrm{CD}^{+}$and $\mathrm{CD} 8^{+}$T-lymphocytes and $\mathrm{CD}_{1}{ }^{+}$B-lymphocytes in peripheral blood and lymph was apoM ${ }^{-/-}$mice, and the quantification of splenic $\mathrm{CD} 4^{+}$ and $\mathrm{CD}^{+}$cells was similar in apoM $^{+/+}$and apoM $^{-/-}$mice without LPS administration. Meanwhile, in our study, we observed that the percentage of splenic CD $4^{+}$T-lymphocytes in the wild type was higher than that in apo $\mathrm{M}^{-/-}$mice before LPS administration. Then, we studied the percentage of $\mathrm{CD}^{+}$ of total lymphocytes and $\mathrm{CD} 4^{+}$of total $\mathrm{CD} 3^{+}$T-lymphocytes in the spleen after LPS administration. We showed that the percentage of $\mathrm{CD}^{+}$and $\mathrm{CD}^{+}$T-lymphocytes was decreased in the spleen after the treatment of LPS. Moreover, we found that even more pronounced decreasing splenic $\mathrm{CD}^{+}{ }^{+}$-lymphocytes occurred in apoM ${ }^{-/-}$mice compared to apoM $\mathrm{M}^{+/+}$mice. Timothy Hla and his colleagues used different strategy of apoM gene knockout model from us $[8,28]$, which was designed to delete $39 \mathrm{bp}$ of the endogenous apoM sequences in exon 2 and insert a neomycin resistanceencoding cassette in the apoM locus. Different apoM gene knockout strategies and experimental design may attribute to these contradictive results in the paper. apo $\mathrm{M}^{+} \mathrm{HDL}$ inhibited the bone marrow proliferation of lymphocyte progenitors, through the activation of S1PR1 on these cells, while the increase of circulating lymphocytes was found in $\mathrm{apoM}^{-/-}$ mice with the exaggerated autoimmune neuroinflammatory response, whereas overexpression of apoM was protective [27]. We speculate that apoM could restrain the bone marrow proliferation of lymphocyte progenitors and the cell cycle of T-lymphocytes from the secondary lymphoid organs to the periphery blood even after the administration of LPS, so the wild type harbored more $\mathrm{CD} 4^{+}$T-lymphocytes in the murine spleen than apoM ${ }^{-/-}$mice. Taking together, our present study may provide certain evidence that apoM might facilitate the maintenance of splenic CD $4^{+}$T-lymphocytes. Moreover, apoM would modify the T-lymphocytes subgroups in the murine spleen, whereas the splenic $\mathrm{CD}^{+}$T-lymphocytes would remain unchanged between apoM $\mathrm{M}^{+/+}$and apoM $^{-/-}$ in these conditions. Thus, it would be of great interest to 
determine the differential effects of apoM on various $\mathrm{CD} 4^{+}$ T-lymphocytes and their subpopulations to gain insights into their roles in different tissue microenvironment.

TNF-alpha, as a member of a pleiotropic inflammatory cytokine, responds to microbes, especially the LPS, and stimulates the acute phase reaction. It is produced chiefly by activated macrophages [29], although it can be produced by many other cell types such as $\mathrm{CD}^{+}$lymphocytes, neutrophils, mast cells, and eosinophils [30]. Monocyte chemotactic protein-1 (MCP-1), also referred to as the chemokine (C-C motif) ligand 2 (CCL2), is a small cytokine that belongs to the CC chemokine family. MCP-1 recruits monocytes, memory $\mathrm{T}$ cells, and dendritic cells to the sites of inflammation $[18,31]$. In the present study, as expected, after LPS treatment, the hepatic mRNA levels and serum protein levels of TNF-alpha and MCP-1 were dramatically increased in both apoM ${ }^{-/-}$mice and apoM ${ }^{+/+}$mice; however, there were no extremely differences found between apoM $\mathrm{M}^{-/-}$mice and apoM $\mathrm{M}^{+/+}$mice. In continuing experiments we will try to investigate other cytokines in the apoM gene deficient mice with or without LPS treatment. It has been reported that high serum levels of endotoxins were found in the patients suffering from common variable immunodeficiency (CVID), which suggest that $\mathrm{CD} 4^{+}$T-lymphocytes dysfunction was restricted to bacteria-specific and not virus-specific $\mathrm{CD} 4^{+} \mathrm{T}$ lymphocytes, including the reduced capacity to proliferate and to produce IFN- $\gamma$ and IL-2 [32]. Persistent inflammation, impaired $\mathrm{CD}^{+}$T-lymphocytes activation, and caused $\mathrm{T}$ lymphocytes exhaustion may be associated with decreased survival in elderly patients and mice after sepsis [33]. Current investigation suggests that T-lymphocytes exhaustion is a newly recognized pathophysiologic mechanism of immunosuppression in sepsis that results in failure to activate macrophages and eradicate invading pathogens, thereby increasing susceptibility to secondary infections, leading to prolonged inflammation [34]. The significant reduction of TNF-alpha after higher dosage of LPS treatment in apoM-1mice might be a partial consequence of the depletion of $\mathrm{CD}^{+}{ }^{+}$T-lymphocytes in the spleen and the exacerbation of inflammatory condition. This finding is in agreement with the reduction of proliferative capacity of $\mathrm{CD}^{+}$memory cells suppressed by persistent antigen presentation extending beyond the priming phase [35].

It is concluded that the present study shows the decrease of splenic $\mathrm{CD}^{+}{ }^{+} \mathrm{T}$-lymphocytes and low ratio of CD4/CD8 in apoM gene deficient mice compared to the wild type, which suggests that apoM might facilitate the maintenance of $\mathrm{CD} 4^{+}$ T-lymphocytes or modify the T-lymphocytes subgroups; it can even accumulate the splenic $\mathrm{CD} 4^{+}$T-lymphocytes after LPS administration in the murine spleen. These results may further explore the importance of apoM on the regulation of immune responses, although the detailed mechanism needs continuing investigation.

\section{Conflict of Interests}

The authors declare that there is no conflict of interests regarding the publication of this paper.

\section{Acknowledgments}

This research project was supported by the National Natural Science Foundation of China (NSFC) (81370372, 81201352) and the Natural Science Foundation of Jiangsu Province (BK2012154). The authors would like to thank Zhu Y. B. and Ma J. B. for excellent technical assistance of flow cytometry analyses.

\section{References}

[1] B. Dahlbäck and L. B. Nielsen, "Apolipoprotein $M-$ a novel player in high-density lipoprotein metabolism and atherosclerosis," Current Opinion in Lipidology, vol. 17, no. 3, pp. 291-295, 2006.

[2] G. Luo, X. Zhang, P. Nilsson-Ehle, and N. Xu, "Apolipoprotein M," Lipids in Health and Disease, vol. 3, article 21, 2004.

[3] J. E. Deakin, A. T. Papenfuss, K. Belov et al., "Evolution and comparative analysis of the MHC Class III inflammatory region," BMC Genomics, vol. 7, article 281, 2006.

[4] C. Wolfrum, M. N. Poy, and M. Stoffel, "Apolipoprotein M is required for pre $\beta$-HDL formation and cholesterol efflux to HDL and protects against atherosclerosis," Nature Medicine, vol. 11, no. 4, pp. 418-422, 2005.

[5] G. D. Norata, A. Pirillo, E. Ammirati, and A. L. Catapano, "Emerging role of high density lipoproteins as a player in the immune system," Atherosclerosis, vol. 220, no. 1, pp. 11-21, 2012.

[6] G. D. Norata, A. Pirillo, and A. L. Catapano, "HDLs, immunity, and atherosclerosis," Current Opinion in Lipidology, vol. 22, no. 5, pp. 410-416, 2011.

[7] A. L. Catapano, A. Pirillo, F. Bonacina, and G. D. Norata, "HDL in innate and adaptive immunity," Cardiovascular Research, vol. 103, no. 3, pp. 372-383, 2014.

[8] C. Christoffersen, H. Obinata, S. B. Kumaraswamy et al., "Endothelium-protective sphingosine-1-phosphate provided by HDL-associated apolipoprotein M," Proceedings of the National Academy of Sciences of the United States of America, vol. 108, no. 23, pp. 9613-9618, 2011.

[9] C. Christoffersen and L. B. Nielsen, "Apolipoprotein M: bridging HDL and endothelial function," Current Opinion in Lipidology, vol. 24, no. 4, pp. 295-300, 2013.

[10] S. Mandala, R. Hajdu, J. Bergstrom et al., "Alteration of lymphocyte trafficking by sphingosine-1-phosphate receptor agonists," Science, vol. 296, no. 5566, pp. 346-349, 2002.

[11] M. Matloubian, C. G. Lo, G. Cinamon et al., "Lymphocyte egress from thymus and peripheral lymphoid organs is dependent on S1P receptor 1," Nature, vol. 427, no. 6972, pp. 355-360, 2004.

[12] G. Liu, K. Yang, S. Burns, S. Shrestha, and H. Chi, “The S1P ${ }_{1}^{-}$ mTOR axis directs the reciprocal differentiation of $\mathrm{T}_{\mathrm{H}} 1$ and $\mathrm{T}_{\text {reg }}$ cells," Nature Immunology, vol. 11, no. 11, pp. 1047-1056, 2010.

[13] N. Xu, X.-Y. Zhang, X. Dong, U. Ekström, Q. Ye, and P. NilssonEhle, "Effects of platelet-activating factor, tumor necrosis factor, and interleukin-1alpha on the expression of apolipoprotein $\mathrm{M}$ in HepG2 cells," Biochemical and Biophysical Research Communications, vol. 292, no. 4, pp. 944-950, 2002.

[14] S. B. Kumaraswamy, A. Linder, P. Åkesson, and B. Dahlbäck, "Decreased plasma concentrations of apolipoprotein $\mathrm{M}$ in sepsis and systemic inflammatory response syndromes," Critical Care, vol. 16, no. 2, article R60, 2012.

[15] X. Ma, Y.-W. Hu, Z.-L. Zhao et al., "Anti-inflammatory effects of propofol are mediated by apolipoprotein $\mathrm{M}$ in a hepatocyte 
nuclear factor-1 $\alpha$-dependent manner," Archives of Biochemistry and Biophysics, vol. 533, no. 1-2, pp. 1-10, 2013.

[16] E. Semaeva, O. Tenstad, J. Skavland et al., "Access to the spleen microenvironment through lymph shows local cytokine production, increased cell flux, and altered signaling of immune cells during lipopolysaccharide-induced acute inflammation," Journal of Immunology, vol. 184, no. 8, pp. 4547-4556, 2010.

[17] S. C. Datta and M. R. Opp, "Lipopolysaccharide-induced increases in cytokines in discrete mouse brain regions are detectable using Luminex xMAP technology," Journal of Neuroscience Methods, vol. 175, no. 1, pp. 119-124, 2008.

[18] J. Zhu and W. E. Paul, "Peripheral CD4 ${ }^{+}$T-cell differentiation regulated by networks of cytokines and transcription factors," Immunological Reviews, vol. 238, no. 1, pp. 247-262, 2010.

[19] T. Kawai and S. Akira, "TLR signaling," Seminars in Immunology, vol. 19, no. 1, pp. 24-32, 2007.

[20] J. P. McAleer and A. T. Vella, "Educating CD4 T cells with vaccine adjuvants: lessons from lipopolysaccharide," Trends in Immunology, vol. 31, no. 11, pp. 429-435, 2010.

[21] E. J. Goetzl, J.-J. Liao, and M.-C. Huang, "Regulation of the roles of sphingosine 1-phosphate and its type $1 \mathrm{G}$ protein-coupled receptor in T cell immunity and autoimmunity," Biochimica et Biophysica Acta (BBA)-Molecular and Cell Biology of Lipids, vol. 1781, no. 9, pp. 503-507, 2008.

[22] A. O. Kamphorst and R. Ahmed, "CD4 T-cell immunotherapy for chronic viral infections and cancer," Immunotherapy, vol. 5, no. 9, pp. 975-987, 2013.

[23] M. Shale, C. Schiering, and F. Powrie, "CD4 ${ }^{+}$T-cell subsets in intestinal inflammation," Immunological Reviews, vol. 252, no. 1, pp. 164-182, 2013.

[24] R. Markwart, S. A. Condotta, R. P. Requardt et al., "Immunosuppression after sepsis: systemic inflammation and sepsis induce a loss of naïve T-cells but no enduring cell-autonomous defects in T-cell function," PLoS ONE, vol. 9, no. 12, Article ID el15094, 2014.

[25] A. J. Wilhelm, M. Zabalawi, J. S. Owen et al., "Apolipoprotein AI modulates regulatory T cells in autoimmune $\mathrm{LDLr}^{-} I^{-}$, ApoA$\mathrm{I}^{-} /{ }^{-}$mice," Journal of Biological Chemistry, vol. 285, no. 46, pp. 36158-36169, 2010.

[26] H. Chi, "Sphingosine-1-phosphate and immune regulation: trafficking and beyond," Trends in Pharmacological Sciences, vol. 32, no. 1, pp. 16-24, 2011.

[27] V. A. Blaho, S. Galvani, E. Engelbrecht et al., "HDL-bound sphingosine-1-phosphate restrains lymphopoiesis and neuroinflammation," Nature, vol. 523, no. 7560, pp. 342-346, 2015.

[28] C. Christoffersen, M. Jauhiainen, M. Moser et al., "Effect of apolipoprotein $\mathrm{M}$ on high density lipoprotein metabolism and atherosclerosis in low density lipoprotein receptor knock-out mice," The Journal of Biological Chemistry, vol. 283, no. 4, pp. 1839-1847, 2008.

[29] C. A. Knosp and J. A. Johnston, "Regulation of $\mathrm{CD} 4^{+} \mathrm{T}-$ cell polarization by suppressor of cytokine signalling proteins," Immunology, vol. 135, no. 2, pp. 101-111, 2012.

[30] K. Świst and E. Pajtasz-Piasecka, "The influence of transcription factors on $\mathrm{CD}^{+} \mathrm{T}$ cell differentiation," Postępy Higieny $i$ Medycyny Doświadczalnej, vol. 65, pp. 414-426, 2011.

[31] K. K. McKinstry, T. M. Strutt, and S. L. Swain, "Regulation of $\mathrm{CD} 4{ }^{+} \mathrm{T}$-cell contraction during pathogen challenge," Immunological Reviews, vol. 236, no. 1, pp. 110-124, 2010.

[32] M. Perreau, S. Vigano, F. Bellanger et al., "Exhaustion of bacteria-specific CD4 $\mathrm{T}$ cells and microbial translocation in common variable immunodeficiency disorders," The Journal of Experimental Medicine, vol. 211, no. 10, pp. 2033-2045, 2014.

[33] S. Inoue, K. Suzuki, Y. Komori et al., "Persistent inflammation and $\mathrm{T}$ cell exhaustion in severe sepsis in the elderly," Critical Care, vol. 18, no. 3, article R130, 2014.

[34] R. S. Hotchkiss, G. Monneret, and D. Payen, "Immunosuppression in sepsis: a novel understanding of the disorder and a new therapeutic approach," The Lancet Infectious Diseases, vol. 13, no. 3, pp. 260-268, 2013.

[35] S. Han, A. Asoyan, H. Rabenstein, N. Nakano, and R. Obst, "Role of antigen persistence and dose for $\mathrm{CD} 4{ }^{+} \mathrm{T}$-cell exhaustion and recovery," Proceedings of the National Academy of Sciences of the United States of America, vol. 107, no. 47, pp. 20453-20458, 2010. 

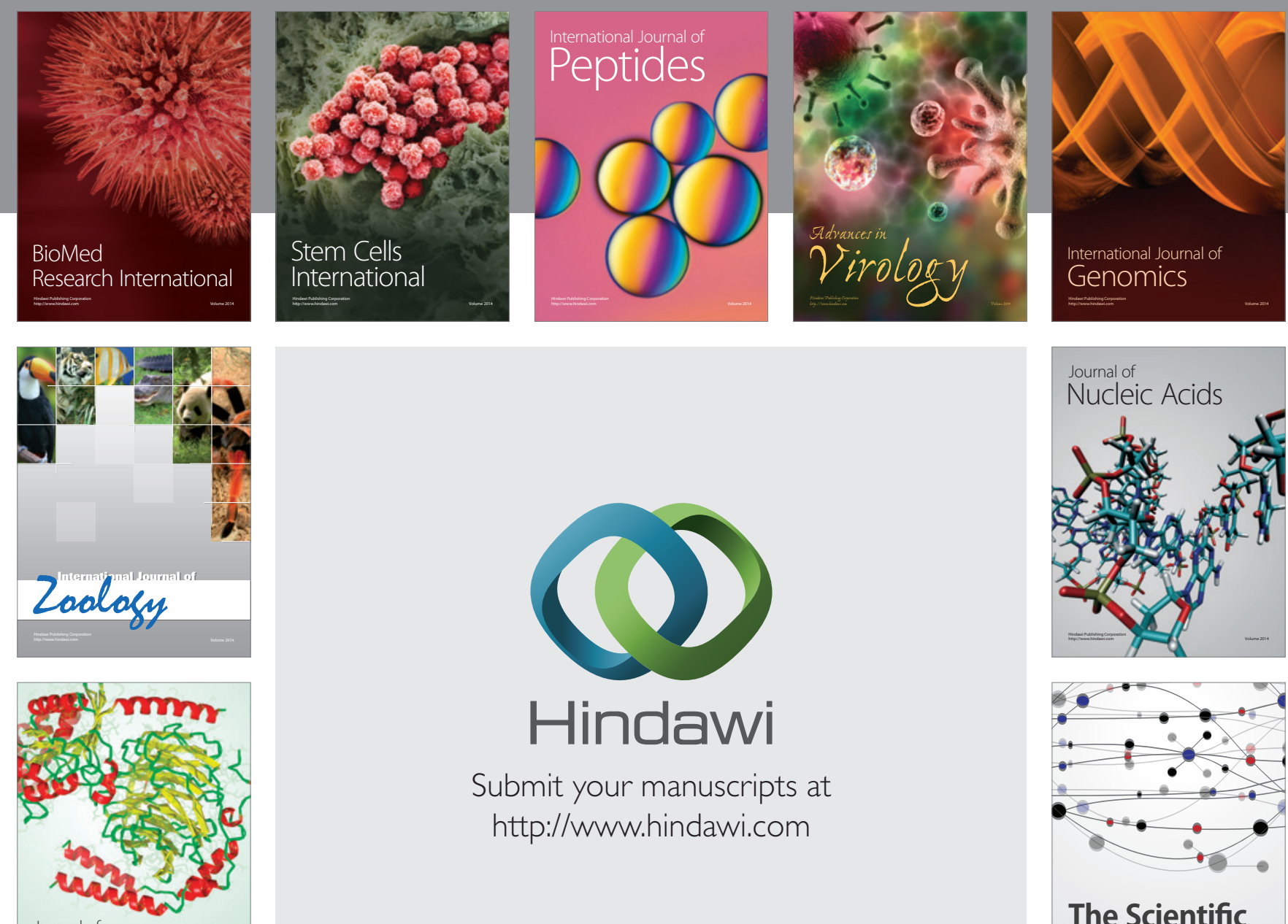

Submit your manuscripts at

http://www.hindawi.com

Journal of
Signal Transduction
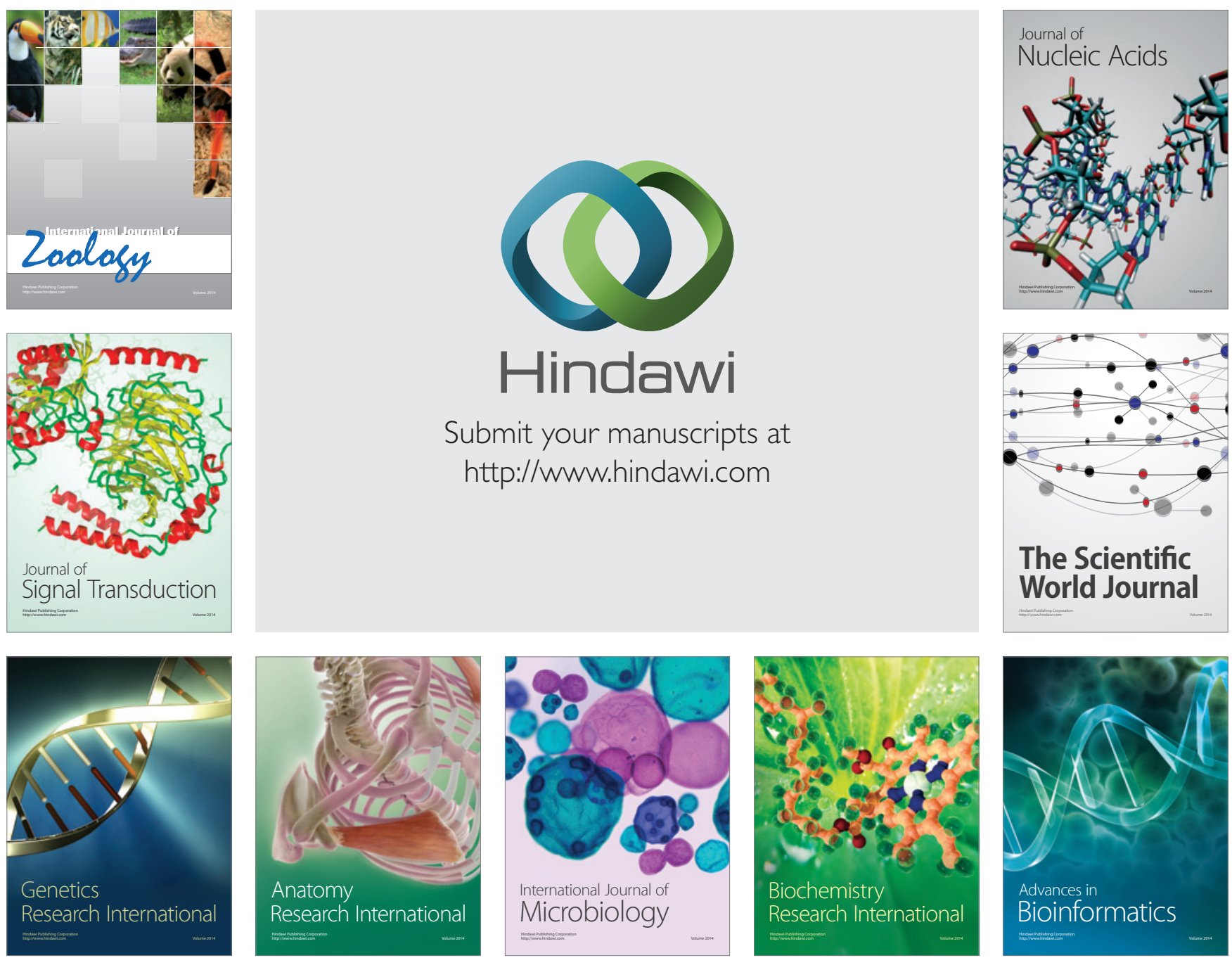

The Scientific World Journal
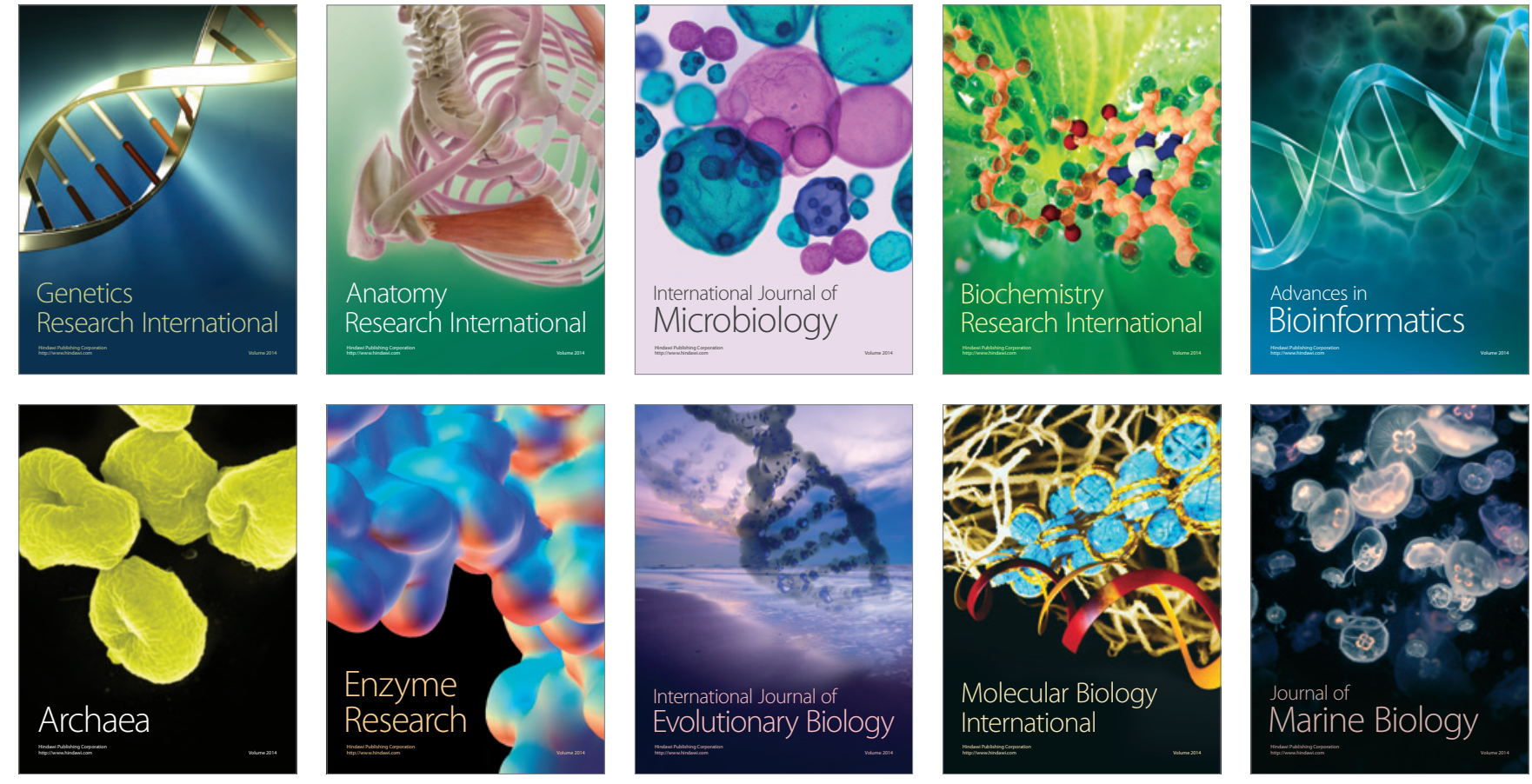